\title{
INCENTIVOS PARA LA INNOVACIÓN: FREEWARE, OPEN SOURCE, Y DERECHOS DE PROPIEDAD INTELECTUAL ${ }^{\circ}$
}

\author{
INCENTIVES FOR INNOVATION: FREEWARE, OPEN \\ SOURCE, AND INTELLECTUAL PROPERTY RIGHTS
}

\author{
Leonardo A. Caravaggio*
}

enviado: 31 agosto 2018 - aceptado: 11 noviembre 2018

\section{Resumen}

El presente trabajo analiza, desde una perspectiva económica, diferentes desafíos tecnológicos que enfrentan los incentivos a la innovación. El freeware y el open source parecieran brindar la respuesta que haga sostenible la industria del software en los próximos años frente al acelerado proceso de posibilidad de copia. Incluso podrían contener respuestas para otras industrias que se enfrentan a una similar situación. Una mejor comprensión de los incentivos será la clave para llevarlo a cabo. El trabajo mira en particular el caso argentino.

Palabras clave: freeware, open source, derechos de propiedad intelectual. Código JEL: O31, D23, L86.

\begin{abstract}
This paper analyzes, from an economic perspective, different technological challenges faced by incentives to innovation. Freeware and open source seem to provide the answer that will make the software industry sustainable in the coming years in the face of the accelerated process of possibility of copying. They may even contain

\footnotetext{
Caravaggio, L. (2020). Incentivos para la innovación: freeware, open source, y derechos de propiedad intelectual. Estudios económicos, 37 (75), 149-163.

* Universidad de Buenos Aires. Correo electrónico: caravaggio@gmail.com
} 
answers for other industries that face a similar situation. A better understanding of the incentives will be the key to carry it out. The work looks in particular at the Argentine case.

Keywords: Freeware, Open Source, Intellectual Property Rights. JEL Code: O31, D23, L86. 


\section{INTRODUCCIÓN}

El esquema de incentivos para el desarrollo de cierto tipo de actividades artísticas e intelectuales parece haber sufrido modificaciones en los últimos años. Los Estados se enfrentan a la posibilidad de acompañar la transición a un nuevo esquema de incentivos o ignorar las nuevas tecnologías intentando proseguir con la implementación de los derechos de propiedad intelectual (DPI) actuales. Prohibir la copia de un disco de pasta o vinilo era una cosa, pero prohibir la copia de un MP3 es prácticamente imposible de llevar a la práctica. La facilidad con que se puede hacer una copia digital de una canción, un disco, un libro, un artículo, una película, una foto, un programa de computadora, hace que esa prohibición sea muy difícil de llevar a la práctica. Y la copia puede ser distribuida con la misma facilidad a través de internet, enviada por mail a una, cien o mil personas, colgada en un servidor de acceso público, o por medio de sistemas de intercambio de información "point to point" (P2P, tipo BitTorrent), u otros.

Hoy en día la posibilidad de copiar y compartir ideas y bienes relacionados con el arte, crea muchas veces a los productores de estos, la imposibilidad de apropiarse de los derechos de propiedad intelectual que en general les corresponden de acuerdo a la legislación. Esto es, como la mayoría de las cosas en la economía, un problema de incentivos. De continuar la presente tendencia, es posible que, a pesar de los esfuerzos de editoriales, discográficas, empresas desarrolladoras de programas de computadora (software), los creadores de estos bienes dejen de producirlos, empiecen a hacerlo por placer, o deban buscar vías alternativas para financiar la producción. De hecho, esto ya sucede, por ejemplo, en el caso de las discográficas que permiten bajarse libremente de internet sus discos, o los escritores que regalan las versiones digitales de sus libros, o en el caso de los programas de computadoras, lo que se conoce como "freeware", esto es un tipo de software que se distribuye en forma gratuita. Sin embargo, probablemente esto los lleve luego a tener que vender entradas de recitales, ediciones "de lujo" en cajas bien presentadas para los libros, o intenten vender la versión "completa" del software, reciban una parte de los ingresos que la página de descarga reciba en concepto de publicidad, o saquen provecho de los datos personales (por ejemplo, la ubicación por GPS) que los usuarios del software aceptan ofrecer (a veces en forma no del todo consiente) a cambio de usarlo.

Otro caso interesante, mucho más difundido todavía, es el de los desarrolladores de software que en lugar de proteger su código lo liberan de manera que otros desarrolladores lo puedan ver o modificar, lo que se conoce como código abierto (open source). Esta forma de colaboración se suele entender como un híbrido entre el modelo de software gratuito, y el de software de pago. Así cómo el freeware puede ser de código 
abierto, o de código cerrado, el open source puede ser distribuido como freeware o como software de pago. Como sea, al permitir a otros acceder al código, el autor decide libremente no reservarse el "derecho de secreto" que el copyright le habilitaría.

Para poder hacer esto se desarrollaron las "licencias libres", como por ejemplo la licencia Creative Commons (CC) bajo la cual se podría permitir el libre intercambio del bien intelectual (software $u$ otro), con lo que se lo convierte en un bien común, de dominio público. No es cierto que los bienes con licencia libre sean de inferior calidad. Muchos de los sistemas operativos basados en UNIX, que son de licencia libre (software libre), son tan o incluso más estables y completos que otros con licencia de código cerrado (EULA), como por ejemplo Windows (algunos llaman a este tipo de programas software privativo, en el sentido de que privan a otros de acceder al código). Tampoco es cierto que nadie vaya a estar dispuesto a trabajar en este tipo de proyectos, incluso sin incentivos pecuniarios de ningún tipo: la enciclopedia más grande de la historia, Wikipedia, se distribuye bajo licencia CC y la mayoría de los colaboradores no reciben un sueldo a cambio de sus aportes (Wikimedia, la organización que lleva adelante, entre otros, el proyecto Wikipedia cuenta solamente con 150 empleados frente a los más de 70.000 colaboradores).

\section{GUERRA POR LOS DERECHOS DE PROPIEDAD INTELECTUAL}

\section{I.1. Movimiento Restrictivo (Pro Copyright): del Estatuto de Venecia al TRIPS}

¿Qué tipo de incentivos son más provechosos para la sociedad y deberían entonces ser apoyados por el Estado? Algunos estudios muestran incluso que no siempre el derecho a monopolio que introducen los DPI aumenta la producción. Por ejemplo, Scherer (2003) lo muestra para el caso de la música clásica y los derechos de propiedad en Europa en los siglos XVIII y XIX. ¿Las leyes que restringen la copia son buenas para la industria?

Los DPI no han sido siempre iguales a lo largo de la historia, ni tampoco lo son hoy entre los distintos países: patentes, prohibición de copia (copyright), registro de marca, etc. De hecho, desde cierta posición intelectual se critica la idea de intentar englobar todos los tipos de restricciones al intercambio de ideas y bienes intangibles bajo la categoría "propiedad intelectual"1. De todas formas, es posible trazar una división de los desarrollos históricos de dos movimientos impulsados

1 http://www.vialibre.org.ar/mabi/1-propiedad_intelectual.htm 
por la evolución de las técnicas de copiado. Uno restrictivo, proteccionista, procopyright, y otro libre, antiproteccionista, anti-copyright.

La imposición de límites legales a la copia está directamente relacionada con la evolución de las técnicas de copiado. En primer lugar, con el desarrollo de la imprenta, y mucho más ahora con la digitalización. Por ejemplo, el Estatuto de Venecia de 1474 puede considerarse el primer intento de protección a la copia, lo que no parece casual a la luz de que el "Misal de Constanza" (el primer libro en ser impreso por una imprenta de tipos móviles) es de 1449. Este estatuto, bajo la autorización de la República, otorgaba un poder de monopolio de 10 años al autor. Con el paso del tiempo, y el desarrollo técnico, la legislación fue ampliando el proteccionismo al creador de la obra. En 1623 Inglaterra establece el Estatuto de Monopolios, el cual excluye de la prohibición monopólica a las patentes. Luego, el Estatuto de la Reina Ana de 1710 fue uno de los primeros intentos en asignarle al autor de una obra escrita derechos sobre su creación que impidan a terceros realizar copias sobre la misma. Una cuestión interesante de este estatuto es que el plazo por el cual se le concedía al autor derechos monopólicos era de apenas 14 años, extensible hasta $28 \mathrm{si}$ el autor continuaba con vida (este plazo fue incrementándose a través de los años). Además de cumplir con el reclamo de autores y editores la Corona se aseguraba así un control sobre lo que se publicaba (Boyle, 2008).

El siguiente hito en la historia de los derechos de propiedad es la Convención de Berna de 1886, a la que la legislación de los distintos países se fue adecuando a lo largo de los años. La idea de este convenio básicamente es extender a los distintos países miembros los derechos que se conceden en uno, de manera que, si tengo patentado un escrito en Inglaterra, tampoco pueda ser copiado en Francia, y así. En su versión actual otorga un derecho de monopolio al autor de la obra durante toda su vida, e incluso a los herederos durante 50 años.

La Organización Mundial de la Propiedad Intelectual (OMPI, o WIPO por sus siglas en inglés), fundada en 1967, se reconoce heredera de la Convención de Berna y del Convenio de París para la Protección de la Propiedad Intelectual de 1883 (más orientado a la propiedad intelectual industrial). Esta organización, perteneciente al sistema de organizaciones de Naciones Unidas (ONU, UN por sus siglas en inglés), es el máximo referente a nivel mundial en lo que respecta a DPI. Administra 26 tratados internacionales entre sus 186 Estados miembro. Se propone como objetivo estimular la innovación y la creatividad ${ }^{2}$.

2 www.wipo.int 
Como otros hechos importantes también debería mencionarse la firma de la Convención Universal sobre Derechos de Autor, adoptada inicialmente en 1952, con versión final en lo que se conoce como Convenio de Ginebra de 1972. También en 1972, se crea la Oficina Europea de Patentes (OEP, o EPO por sus siglas en inglés), otro importante organismo que sigue dando forma al proceso de profundización e internacionalización de los DPI.

Como legado de estas organizaciones y el proceso de internacionalización de los DPI, con fuerte impulso de los Estados Unidos y bajo administración de la Organización Mundial del Comercio (OMC, WTO por sus siglas en inglés) se firma en 1994 el Acuerdo sobre los Aspectos de los Derechos de Propiedad Intelectual relacionados con el Comercio (ADPIC, o TRIPS por sus siglas en inglés). Este es un tratado que pone más énfasis en lo referente a la propiedad intelectual industrial, pero no puede dejar de ser mencionado por su relevancia, y la extensión que hizo a todos los países miembro de la OMC.

Los más recientes avances en esta línea son los referentes al desarrollo de los derechos digitales, y las medidas técnicas de protección efectiva. Este tipo de desarrollos normativos buscan regular la distribución de las ideas a través de internet.

\section{I.2. Movimiento libertario (anti-copyright): de Gutenberg al MP3}

En paralelo a este desarrollo, fue creciendo también un movimiento con otros incentivos que abogaron por una liberalización de los derechos de propiedad. Los principales argumentos de este tipo de posición es el del beneficio del mayor número de personas, el hecho de que los bienes en cuestión sean (en la mayoría de los casos) bienes públicos (no rivales y no excluibles) y, en ese sentido, que el costo marginal de agregar una unidad (hacer una copia) sea igual a cero (Boyle, 2008). O, al menos, cada vez más bajo con cada nuevo desarrollo del proceso técnico de copia.

A un nivel filosófico-ético-moral, las críticas al proceso de desarrollo de los DPI son tan antiguas como los derechos mismos. Sin embargo, no están tan bien documentados, e incluso algunos autores, desde una posición bastante fundamentalista, plantean que la historia es reescrita de modo de justificar el capitalismo liberal, que se desarrolla primero en base a libre comercio, y luego impone barreras para que los demás no puedan desarrollarse (patean la escalera). El ejemplo más comentado en este sentido es el de Holanda que desarrolló fuertemente su industria a pesar de haber abolido las patentes en 1869, y haberlas restaurado recién en 1912, sin que esto se proponga como ejemplo a seguir (Chang, 2010). 
Mientras más fuerte se volvió el proceso pro-copyright, más fuertes se volvieron los movimientos anti-copyright. Mientras más sencillo hizo el avance de la tecnología la posibilidad de realizar copias, más fueron necesarias leyes y programas concientizadores pro-copyright (como por ejemplo los llevados adelante por la Business Software Alliance), y a su vez más reclamaron los movimientos anti-copyright que no existan leyes que prohíban la copia y difusión de ideas (música, libros, software, etc.).

El primer movimiento realmente fuerte en este sentido puede asociarse a la década del ochenta. Con el desarrollo de la computación y el ingreso de cada vez mayor número de personas a este mundo, algunos programadores a los que luego se conocería como "hackers" hicieron un avance en pos de la liberación de la información. Si bien muchas veces se confunde a los hackers con destructores de información (crackers), el espíritu original de esta cultura está vinculado a personas con altos conocimientos de informática que buscaban socializar, hacer accesible a todos, el software y la información digital. Aun así, no siempre lo hicieron dentro del marco estricto de la legalidad, sino muchas veces copiando programas, vulnerando sistemas de seguridad informáticos, etc.

Sin embargo, la verdadera revolución de estos movimientos se dio recién en la década del noventa. Y continuó desarrollándose desde entonces, a tal punto que hoy en día es difícil identificar a los cientos de expresiones en esta línea bajo un mismo, o similar reclamo. Solo por poner algunos ejemplos, podemos mencionar a Piratbryan, Coloriuris, GNU, Copyleft, Pirate Cinema, etc. Todos estos son grupos de personas que abogan por la libertad a la hora de compartir y copiar música, libros, software, películas, y todo lo que se pueda. Incluso algunos se han organizado como partidos políticos, como el Piratpartiet de Suecia, que dio origen a los distintos "Partidos Pirata" alrededor del mundo 3 . O incluso han encontrado una mejor forma de organización como religión, tal es el caso del Kopimismo (de forma que bajo el pretexto de la libertad de culto copian y comparten información).

Un movimiento en particular que vale la pena destacar es que el que surge en 1998 como respuesta al acta Sonny Bono de extensión de los términos de Copyright. Esta ley pretendía extender los derechos de autor en la legislación de Estados Unidos. Frente al debate sobre este proyecto, Lawrence Lessing se hace famoso por su oposición a la reforma, y luego por llevar el caso a la corte. Pierde el caso en 2001, pero sin embargo, motivado por esta tarea, funda la organización "Creative

3 El partido pirata tiene representación en Argentina: http://partidopirata.com.ar/ 
Commons" una de las organizaciones más fuertes en la lucha anti-copyright, y creadora de las licencias CC.

En contraposición al "todos los derechos reservados" que acompaña a las leyendas de copyright, se suele acompañar a las leyendas de licencias CC con la frase "algunos derechos reservados", haciendo alusión a las cosas que ese tipo de licencias permite hacer con el bien reservado (por ejemplo, el permiso de ver, copiar o modificar el código fuente). ¿Es viable el desarrollo de ese tipo de iniciativas? ¿Cuándo y cómo se produce la compensación económica? ¿Puede hacerse algo bueno sin compensación económica a cambio?

\section{INCENTIVOS Y DERECHOS DE PROPIEDAD INTELECTUAL}

II. 1. Viabilidad de los modelos con "algunos derechos reservados"

Para quienes buscan darle mayor difusión a su trabajo, los DPI pueden convertirse en una barrera. Así es como algunas discográficas, generalmente pequeñas, deciden permitir que sus discos sean descargados gratuitamente por internet, lo mismo que algunos productores de películas, escritores de libros, etc. También muchas universidades están usando las licencias CC para dar difusión a sus trabajos ${ }^{4}$. Esta actitud genera efectos en cadena ya que permite a un número mucho mayor de personas tener acceso a esas investigaciones y esos conocimientos. $Y$ la retroalimentación de este sistema puede también pensarse como un incentivo a liberar los derechos de lo publicado.

Una fuerte crítica en torno a la liberación de los DPI es que sin ellos no existirían los incentivos suficientes para que las personas dediquen su esfuerzo, talento, y dinero al desarrollo de los bienes intelectuales. Esto es: una farmacéutica, por ejemplo, destina millones de dólares y muchos años de investigación al desarrollo de una nueva droga, y sin una ley que le asegure el derecho de monopolio sobre la venta del remedio vinculado a esa droga, no estaría dispuesta a invertir. Lo mismo sucede en el software: diseñar un sistema operativo (SO) lleva años del trabajo de un importante número de ingenieros altamente capacitados. Si bien esto suena lógico, la realidad demuestra que muchos proyectos se llevan adelante sin estos incentivos. Recientemente, GlaxoSmithKline, una de las empresas farmacéu-

4 Por ejemplo, la Universidad de Oxford tiene una sección donde ofrece trabajos bajo licencia CC. http://podcasts.ox.ac.uk/open 
ticas más grandes del mundo, licenció todos sus componentes contra la Malaria bajo licencia $\mathrm{CC} 0$ (sin derechos reservados), convirtiéndolas en dominio público ${ }^{5}$. En el campo de la informática los ejemplos son mucho más comunes.

Wikipedia, la mayor enciclopedia de la historia, cuenta con más de 37 millones de artículos en 284 idiomas $^{6}$. Es editada colaborativamente desde 2001, y se distribuye bajo licencia CC. Es una de las páginas web más consultadas del mundo. Cualquiera con acceso puede editar los artículos, esto provoca que existan muchas críticas respecto de la parcialidad y la posibilidad de ser atacada con información falsa o eliminando contenido. Sin embargo, estudios muestran que el vandalismo es rápidamente reparado, y el sistema de registro de modificaciones permite recuperar la información si fuera eliminada (Viégas et al., 2004). De hecho, cada vez más, aparece Wikipedia como una fuente de consulta citada en artículos académicos. Esto plantea todo un debate aparte sobre la democratización de la construcción del conocimiento (Pons, 2008). Como sea, la cantidad y calidad de los artículos demuestra que no faltan incentivos para la producción del contenido.

Linux es el kernel del sistema operativo libre y de código abierto más famoso del mundo. Aunque muy lejos de Windows, incluso lejos del SO de Mac, Linux es el tercer SO más usado del mundo, lo que no es poca cosa teniendo en cuenta que fue desarrollado por colaboradores de todo el mundo y se distribuye mediante una licencia pública (GNU General Public License, de la Free Software Fundation). El proyecto fue iniciado por Linus Torvalds en 1991, quien en 2009 quedó fuera de la lista de los treinta desarrolladores que más modificaciones le hacen al sistema (Kroah-Hartman et al., 2009). Esto es curioso porque quiere decir que el proyecto excede su control: no es algo que él por sus medios lleve adelante, sino que hay muchos colaboradores más importantes que él mismo. Torvalds piensa que los incentivos de estos programadores no están en la fama o la reputación, sino más bien en el simple placer de programar. En ese sentido, son como artistas (Ghosh, 1998).

Pero también muchos artistas, los músicos en particular, tienen costos y luchan (muchos de ellos) junto a las discográficas contra la piratería y a favor del copyright. Otros, por el contrario, avanzan por la línea de la liberalización de su producción. El álbum Ghost I-IV de Nine Inch Nails (NIN) se distribuye bajo licencia CC, y fue nominado en 2008 a dos premios Grammy. El disco fue producido por Interscope Records, una submarca de Universal Music. La modalidad de licencia

5 http://wiki.creativecommons.org/Case_Studies/GlaxoSmithKline

$6 \mathrm{http}: / /$ wikipedia.org 
CC que se usó autoriza a terceros a modificar o usar el material para propósitos no comerciales, siempre y cuando mantengan la licencia $\mathrm{CC}$ sobre el nuevo producto. Esto permitiría, entre otras cosas, a nuevos artistas a hacer uso del mismo para la producción de los cada vez más difundidos "mash up's" (producir una nueva canción a partir de la mezcla de dos o más canciones). Trent Reznor, por entonces líder de NIN, justificó el uso de este tipo de licencia señalando que "sintieron que eso es lo apropiado", y que "los derechos de propiedad están anticuados" (Davis, 2008). El álbum fue vendido o descargado más de 750 mil veces reportando ganancias de más de 1.6 millones de dólares.

La mayor parte de internet hoy en día se basa en software open source. Más del $60 \%$ de los servidores web son Apache, open source. Uno de los servidores de correo para el envío y recepción de mails más conocido es Sendmail, que también es open source, al igual que el navegador Firefox, uno de los más usados del mundo. El Google Chrome, otro importante navegador, tiene ciertos frames que también son open source. Estos dos navegadores, además, se distribuyen en forma gratuita.

Estos ejemplos fueron seleccionados para mostrar que existe otro tipo de incentivos, que no son los directamente pecuniarios, que surgen de la venta de licencias y la protección de los desarrollos intelectuales bajo normas estrictamente proteccionistas, que de todas formas son capaces de generar proyectos incluso de gran envergadura. Ahora bien: ¿vale la pena apoyar el desarrollo de este tipo de software? ¿El modelo de negocio que plantean es mejor que el "tradicional"? ¿Cuáles son las ventajas?

\section{II.2. Comparación entre ambos modelos. Incentivos subyacentes}

El modelo con limitados derechos de propiedad intelectual no solo es viable, sino que ha dado muestras de lograr elaboraciones de gran calidad. Muchos incluso creen que para algunos bienes es preferible este modelo, y el ejemplo más común de esto es el software. Los programas open source son elaborados por un número mucho mayor de programadores que los "programas propietarios" (de código cerrado), esto reduce la probabilidad de errores, porque hay más personas revisando y evaluando el código. Esto se conoce como "Ley de Linus" (en honor a Linus Torvalds).

La primera distinción que es necesario hacer en torno a los desarrolladores open source es entre los desarrolladores individuales y los desarrolladores que son parte de una empresa (López, 2003). Aproximadamente un 20\% de las modificaciones aportadas al kernel de Linux provienen de programadores individuales; el 
resto, directamente de empresas que dedican sus recursos a este fin, siendo las más importantes RedHat (12\%), IBM (7\%) y Novell (7\%) (Kroah-Hartman et al., 2009). Claramente los incentivos de unos y otros no son los mismos. En el primero de los casos, los programadores no pueden obtener beneficios económicos, de hecho, muchas veces sus aportes son anónimos. En el segundo de los casos es más probable la existencia de actividades complementarias que permitan a las empresas recuperar los costos de inversión en desarrollo de software open source. Por ejemplo, el recupero mediante cuotas de mercado, intentando establecer un determinado modelo vía open source (más barato y de mayor alcance) hasta convertirlo en un estándar de mercado al que se le puede sacar provecho por determinadas razones. La otra vía es la de RedHat que se vale de las cláusulas de la licencia que le permiten hacer uso comercial del software modificado.

\section{ALTERNATIVAS DE REGULACIÓN. ROL DEL ESTADO}

Una de las funciones del Estado, no siempre aprovechada, es la de acompañar (acelerar) procesos que se darían por sí solos, pero que en su carácter de buenos para la sociedad es conveniente impulsarlos. Si se adhiere a la idea de que el software abierto es una alternativa ventajosa para la sociedad, el Estado podría entonces impulsarlo. Una manera de hacerlo es mediante la compra pública, o el desarrollo de software tipo open source, esto es: recomendar, u obligar a los organismos públicos que compren o desarrollen este tipo de software, por ejemplo, Linux en vez de Windows. Iniciativas de este tipo fueron llevadas adelante por ejemplo por la Junta de Extremadura en España, o a mayor escala por el estado de Rio Grande do Sul, en Brasil. Este último caso impulsó al gobierno federal a llevar adelante un proyecto de viraje hacia el software libre.

En Argentina, hay varios intentos en esta línea. La Secretaría de Hacienda del Ministerio de Economía y Finanzas Públicas lleva adelante un proyecto de software libre para las operaciones transaccionales. Este software, que se denomina e-sidif, incluye cada vez a un mayor número de organismos y tiene entre otras ventajas una reducción en los costos de desarrollo e implementación por su carácter de open source 7 . Otro ejemplo es el programa con el que se hacen los pasaportes Argentinos, que pertenece a la empresa Red Hat y es también open source ${ }^{8}$. O el SO Huayra, basado en Debian GNU/Linux, desarrollado por Anses como parte del

7 http://administracionfinanciera.mecon.gov.ar/

8 Sombreros rojos para la administración pública (martes 26 de octubre de 2010). Página 12. Cultura Digital. Recuperado de https://www.pagina12.com.ar/diario/cdigital/31-155680-2010-10-26.html 
programa Conectar Igualdad. Este SO estará disponible para todas las Netbooks del programa y también para ser descargado gratuitamente, quedando el código como propiedad del Estado nacional argentino 9 .

El otro gran marco de intervención estatal posible es por medio de la regulación. En este sentido, es muy interesante el estudio de los efectos en innovación después del ingreso al Trips. Argentina, como miembro de la OMC, ingresó al Trips en 1995. En particular una discusión en ese sentido se da en torno al uso de patentes para software. En Argentina el software no se patenta (se puede registrar, pero no patentar), como así tampoco en la mayoría de los países de América Latina, aunque sí en Japón o Estados Unidos. No queda claro si esta legislación local es compatible o no con Trips. Desde una posición más proteccionista se concluye que esto no se ajusta a Trips y que sería preferible permitir el patentamiento de software, mientras que desde una posición más libertaria se postula todo lo contrario. Si se permite el patentamiento, y se toman las medidas necesarias para evitar las copias piratas, se favorece la apropiación de los beneficios en forma directa, lo que puede resultar de incentivo para que empresas de software (por ejemplo) se radiquen en el país. Sciancalepore (2007) lleva adelante un estudio pormenorizado y relativamente actualizado del caso Argentino.

Finalmente, el Estado suele financiar proyectos de desarrollo de software libre. Ya sea directamente, o por medio de universidades, cámaras o federaciones de empresas desarrolladoras de software. Por ejemplo, en Argentina, el Ministerio de Ciencia, Tecnología e Innovación Productiva (MinCyT) lleva adelante el Fondo Tecnológico Argentino, que financia inversiones en software libre por medio de la Cámara Argentina de Empresas de Software Libre (CADESOL) y la Universidad de Entre Ríos (UNER) ${ }^{10}$. En los últimos años también se desarrolló un portal de la Secretaría de Modernización que busca facilitar el acceso a los datos públicos (información sobre ingresos y gastos, población, transporte, etc.). Bajo la idea de que estos datos le pertenecen a los ciudadanos, se busca la forma de hacerlos más accesibles y fáciles de aprovechar ${ }^{11}$.

9 http://huayra.conectarigualdad.gob.ar/

$10 \mathrm{http} / / /$ www.mincyt.gob.ar/

11 http://datos.gob.ar/ 


\section{CONCLUSIONES}

Es innegable que no solo los niveles tecnológicos alcanzados, sino también la velocidad de estos cambios, hacen y van a seguir haciendo cada vez más fácil reproducir un número creciente de cosas. Este es un proceso que se inició hace mucho tiempo, pero continúa, se profundiza y acelera. Por otro lado, el proceso legal de restricción de la copia y protección de los derechos del autor es tan antiguo como el otro, pero para resistir debería seguir profundizando y haciendo más fuerte su restricción.

La identificación de los incentivos de los desarrolladores de software open source y freeware no es sencilla. Cada desarrollador o grupo de desarrolladores persigue incentivos propios. De esta forma, reconocemos incentivos pecuniarios - como los de las grandes empresas que solo usan el open source como plataforma de lanzamiento, o el intento de reducción de costos por parte del Estado o algunas empresas-, hasta llegar a los más altruistas, como por ejemplo los programadores y colaboradores que trabajan ad honorem realizando aportes desde todas partes del mundo a grandes proyectos informáticos como Linux o Wikipedia. Dichos incentivos se ven atravesados por las motivaciones más de tipo filosófico-morales, vinculadas al compartir, lo que incluye la concepción de las mejoras que esto implica, como por ejemplo la "Ley de Linus" o la retroalimentación en los procesos de investigación (hasta quienes consideran el compartir como su religión).

El desarrollo de las licencias de software libre (tipo GNU GPL), o de copyright libre (tipo CC), es en buena medida un mecanismo de reacción frente al desarrollo tecnológico que permite el cada vez más sencillo proceso de copia. Y, por otro lado, es producto de los fuertes incentivos que están detrás de cierto tipo de desarrolladores que incluso buscan la forma de copiar legalmente, distribuir y permitir modificar sus producciones.

El Estado (en particular, el Estado argentino) no es ajeno a este proceso y ya está participando activamente del mismo. Por un lado, de forma enérgica en el proceso creador de open source (Huayra y el e-sidif) o la compra de productos desarrollados por otros (como el software que permite producir los pasaportes argentinos). Por otro lado, mediante leyes que obliguen a los distintos niveles del Estado a la adquisición de este tipo de software (como el sector Público de Río Negro $^{12}$, Argentina Ley Provincial 4747, promulgada el 9 de abril de 2012) o de

12 http://www.legisrn.gov.ar 
distintos tipos de invitaciones e incentivos (los casos de Extremadura y Rio Grande do Sul). También hay ejemplos de subsidios directos por parte del Estado a organizaciones que buscan el desarrollo de software libre, como manera de incentivarlo (por ejemplo, desde el MinCyT). Resta preguntarse si esto no es contradictorio con otros procesos pro-copyright en avance (como la participación en Trips).

El freeware y el open source son dos mecanismos de desarrollo de software más presentes de lo que podría parecer. Es posible que contengan en su forma la respuesta que haga sostenible la industria en los próximos años frente al acelerado proceso de posibilidad de copia. Incluso podrían contener respuestas para otras industrias que se enfrentan a similar situación. Una mejor comprensión de los incentivos será la clave para llevarlo a cabo.

\section{REFERENCIAS BIBLIOGRÁFICAS}

Bell, M. (2009). Innovation Capabilities and Directions of Development. STEPS Working Paper No. 33, Brighton: STEPS Centre.

Boldrine, M., \& Levine, D. (2008). Perfectly competitive innovation. Journal of Monetary Economics, 55 (3), 435-453.

Boyle, J. (2008). The Public Domain. Enclosing the commons of the mind. New Haven London: Yale University Press.

Chang, H. (2010). 23 things they don't tell you about capitalism. New York: Bloomsbury Press.

Chang, H. (2002). Kicking away the ladder. Development strategy in historical perspective. London: Anthem Press.

Corat, B., \& Weinstein, O. (2011). Patent Regimes, Firms and the Commodification of Knowledge. Socio-Economic Review, 10 (2), 267-292.

Davis, K. (2008). Potent Quotables: Reznor's Release Strategy. Spinner.com. AOL. 04 de Mayo de 2005. Recuperado de https://goo.gl/aPJBYu

Fink, C., \& Maskus, K. (2005). Intellectual Property and development. Lessons from recent Economic research. Washington, DC: World Bank y Oxford University Press.

Ghosh, R. A. (1998). FM interview with Linus Torvalds: what motivates free software developers? First Monday: Peer-Reviewed Journal on the Internet. Recuperado de https://firstmonday.org/ojs/index.php/fm/article/view/583/504

Johns, A. (2009). Piracy: The intellectual property rights wars from Gutemberg to Gates. Chicago: The University of Chicago Press.

Kroah-Hartman, G., Corbert, J., \& McPherson, A. (2009). Linux Kernel Development How Fast it is Going, Who is Doing It, What They are Doing, and 
Who is Sponsoring It: An August 2009 Update. The Linux Fundation. Recuperado de https://www.linuxfoundation.org/events/2016/08/linux-kerneldevelopment-2016/

Leser, W. (2012). Measuring Intellectual Property 'Strength' and Effects: An Assessment of Patent Scoring Systems and Causality The Journal of Business, Entrepreneurship and the law, 4 (2), 345-382

López, A. (2003). Propiedad Intelectual y nuevas tecnologías. Trabajo presentado en la Reunión Regional de expertos sobre el Sistema Nacional de Innovación: Propiedad Intelectual, Universidad y Empresa organizada por OMPI-CEPAL en Santiago de Chile, 1 al 3 de Octubre de 2003. Recuperado de https://www.wipo.int/ edocs/mdocs/sme/es/ompi_cepal_inn_san_03/ompi_cepal_inn_san_03_t4_3.pdf

Pons, A. (2008). Internet: un reto para el conocimiento (histórico). Pasajes Revista de pensamiento contemporáneo, 27, 30-43

Schwarzman, S. (2008). Universidades y desarrollo en Latinoamérica: experiencias exitosas de centros de investigación. Unesco-Iesalc. Recuperado de http://www.iesalc.unesco.org.ve/dmdocuments/biblioteca/libros/Universidad_ydesarrollo_en_latinoamerica_Version_\%20completa.pdf

Scherer, F. M. (2003). Quarter Notes and Bank Notes: The Economics of Music Composition in the Eighteenth and Nineteenth Centuries. Princeton University Press, Princeton.

Sciancalepore, M. E. (2007). Protecting New Technologies in Latin America: The Case for Computer Software Patents in Argentina. The University of Miami Inter-American Law Review, 38 (2), 349-404.

Viegas, F., Wattemberg, M., \& Kushal, D. (2004). Studying Cooperation and Conflict between Authors with history flow Visualizations. Chi, 6 (1), 575-582

(C) 2020 por los autores; licencia no exclusiva otorgada a la revista Estudios económicos. Este artículo es de acceso abierto y distribuido bajo los términos y condiciones de una licencia Atribución-No Comercial 4.0 Internacional (CC BY-NC 4.0) de Creative Commons. Para ver una copia de esta licencia, visite http://creativecommons.org/licenses/by-nc/4.0 
